\title{
MÉTODOS GEOFÍSICOS PARA LA PROSPECCIÓN, EXPLORACIÓN Y EVALUACIÓN DE MINERAL DE HIERRO
}

\author{
(Iron Ore Mineral's Evaluation, Exploration and Prospection \\ by Geophysical Methods)
}

\section{Sandra Marcela Castro Gaitán, Gehovell Yadira Rodríguez Vega}

Escuela de Ingeniería Geológica, Uptc-Sogamoso

sandrukiss20@yahoo.es -gehovellyadira@yahoo.com

(Recibido mayo 15 de 2006 y aceptado febrero22 de 2007)

\section{Resumen:}

Expone los resultados obtenidos en la prospección y exploración de mineral de hierro en una localidad del municipio de Firavitoba (Boyacá-Colombia), con el objeto de definir la continuidad del material. Se seleccionaron tres métodos geofísicos: Magnetometría, Sondeo Eléctrico Vertical y Cuerpo Cargado Una vez que los datos obtenidos fueron procesados en el software SURFER 8 e IPI2WIN, se obtuvo el mapa de anomalía magnética, el perfil geológico geofísico del área y el mapa de isohomas, de cuya interpretación se definió la continuidad del mineral, la profundidad aproximada a la cual se encuentra el techo de mineralización y el sector más apropiado para la explotación con miras a iniciar una minería a gran escala.

Palabras clave: Magnetometría, SEV, Cuerpo Cargado.

\section{Abstract:}

The outcomes in an iron ore mineral prospection and exploration, made in the municipality of Firavitoba (Boyacá-Colombia) are exposed, with the aim to define the material continuity. Three geophysical methods were selected: The Magneto-metric, the Vertical Electrical Sounding and The Body Loaded. Once these studies were processed in the software SURFER 8 and IPI2WIN, was obtained: The magnetic anomaly map; the area's geophysical geologic profile and an isohomas map. Their interpretation define the mineral continuity, the mineralization roof's approximate depth, and which is the most appropriate sector for exploitation with the eyesight toward a great scale mining's beginning.

\section{INTRODUCCIÓN}

$\mathbf{L}$ os métodos geofísicos resuelven ciertos problemas geológicos apoyándose en el estudio de campos físicos naturales o artificiales y se engloban dentro del conjunto de las ciencias geológicas como una herramienta auxiliar en la prospección y exploración de los yacimientos minerales.

Hace unos años en la vereda San Antonio, del municipio de Firavitoba, en el departamento de Boyacá, se realizaron algunas excavaciones para la búsqueda de mineral de hierro, que fueron abandonadas al no encontrar continuidad del mineral, perdiendo toda la inversión que se había realizado. Esta situación se habría podido evitar utilizando el mismo capital en estudios de prospección por medio de métodos geofísicos de superficie.

El presente proyecto busca destacar la aplicabilidad que tienen tres métodos geofísicos en la localización del sector más apropiado en zona de estudio para explotación de mineral de hierro con miras a iniciar una minería a gran escala.

\section{LOCALIZACIÓN}

El área de estudio se encuentra situada en la vereda San Antonio, municipio de Firavitoba, al centro-oriente del depar- tamento de Boyacá, a una distancia de $9 \mathrm{~km}$ de la ciudad de Sogamoso, capital provincial (ver figura 1). Comprende las coordenadas X: $1.110 .780 \mathrm{Y}: 1.115 .060$ y X: $1.111 .125 \mathrm{Y}$ : 1.115.344 y un área total de $4000 \mathrm{~m}^{2}$.

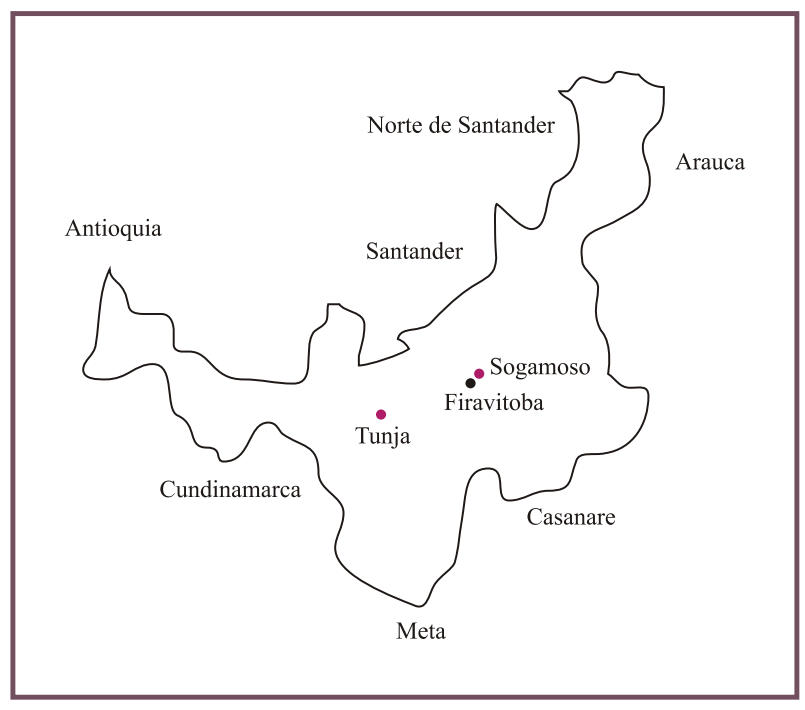

Figura 1. Localización de la zona de estudio. 


\section{METODOLOGÍA}

En la ejecución del proyecto se realizó el siguiente conjunto de actividades:

- Recopilación bibliográfica.

- Levantamiento topográfico del área a escala 1:1000, con el planteamiento de una malla de 20x20 metros.

- Muestreo del mineral, del cual se elaboró una probeta pulida y una sección dejada.

- Levantamiento magnetométrico a escala 1:1000 con los magnetómetros de fabricación canadiense.

- Levantamiento geoeléctrico mediante los métodos sondeo eléctrico vertical y cuerpo cargado.

- Procesamiento de los datos con software especializado (Surfer 8, Ipi2Win).

- Elaboración de modelos geológicos-geofísicos e interpretación de estos.

\section{RESULTADOS}

Los logros obtenidos a través de la ejecución del proyecto fueron:

\subsection{Análisis petrográfico}

A través del microscopio y el contador puntual se establecieron los porcentajes de minerales que constituyen la manifestación de hierro tanto en sección delgada como en la probeta pulida. En la figura 2 se muestra una imagen de la probeta pulida con un aumento del objetivo de 50X, y en la tabla 1 se consigna el resumen del análisis petrográfico realizado a esta; de igual manera, la figura 3 es una imagen de la sección delgada con su correspondiente análisis mostrado en la tabla 2.

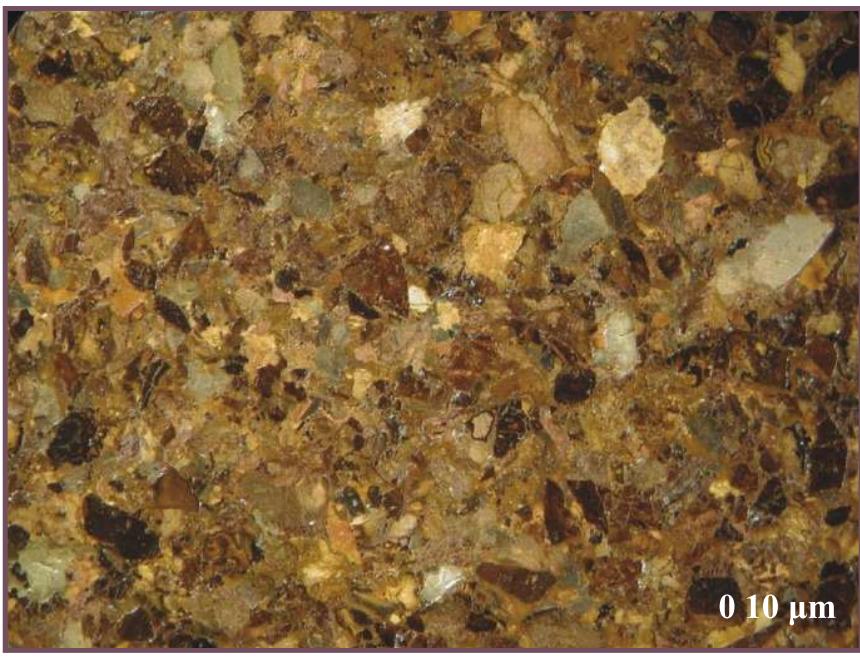

Figura 2. Probeta pulida vista al microscopio.
Tabla 1. Análisis petrográfico probeta pulida.

\begin{tabular}{|l|}
\hline Aumentos del objetivo: $50 \mathrm{X}$ \\
\hline Textura: Clástica \\
\hline Posición del nicol: Paralelo \\
\hline Minerales presentes en orden de abundancia: $52 \%$ Gohetita \\
$32 \%$ Limonita \\
$16 \%$ Arcilla
\end{tabular}

Tabla 2. Análisis petrográfico sección delgada.

\begin{tabular}{|l|}
\hline Aumentos del objetivo: $50 X$ \\
\hline Textura: Clástica \\
\hline Posición del nicol: Paralelo \\
\hline Minerales presentes en orden de abundancia: $\begin{array}{l}51,6 \% \text { Gohetita } \\
31 \% \text { Limonita } \\
14,4 \% \text { Arcilla } \\
3 \% \text { cuarzo }\end{array}$ \\
\hline Origen: Sedimentario \\
\hline Clasificación: Roca ferruginosa \\
\hline
\end{tabular}

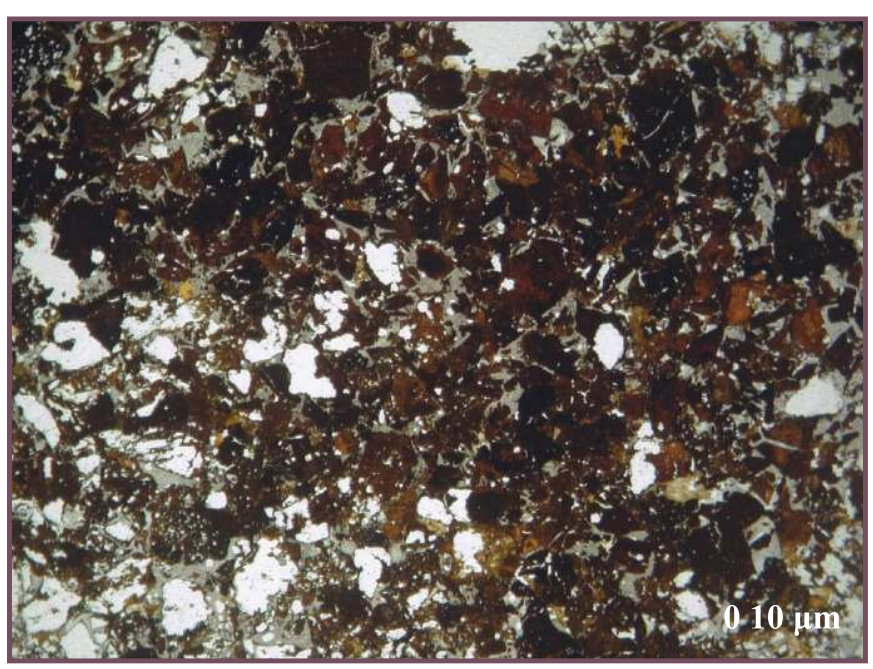

Figura 3. Sección delgada vista al microscopio.

Del anterior reporte se puede determinar que el porcentaje de minerales de hierro es significativo (83\%). Este reporte se realizó a muestras expuestas a la meteorización, por lo que se espera que a profundidad la calidad sea aún mayor y el prospecto de esta mena aumente. 


\subsection{Prospección geofísica}

La prospección geofísica se llevó a cabo en tres etapas: la primera correspondió a la investigación, usando el método magnetométrico; la segunda, al método del cuerpo cargado, y finalmente se aplicó el método del sondeo eléctrico vertical (SEV). En la figura 4 se observa el plano de exploración geofísica; en él se muestra un mallado con 99 puntos que van a ser levantados en el $1 .^{\text {er }}$ y $2 .^{\text {do }}$ método, con su correspondiente propiedad física (campo magnético y resistividad), y en color rojo la ubicación de los SEV.

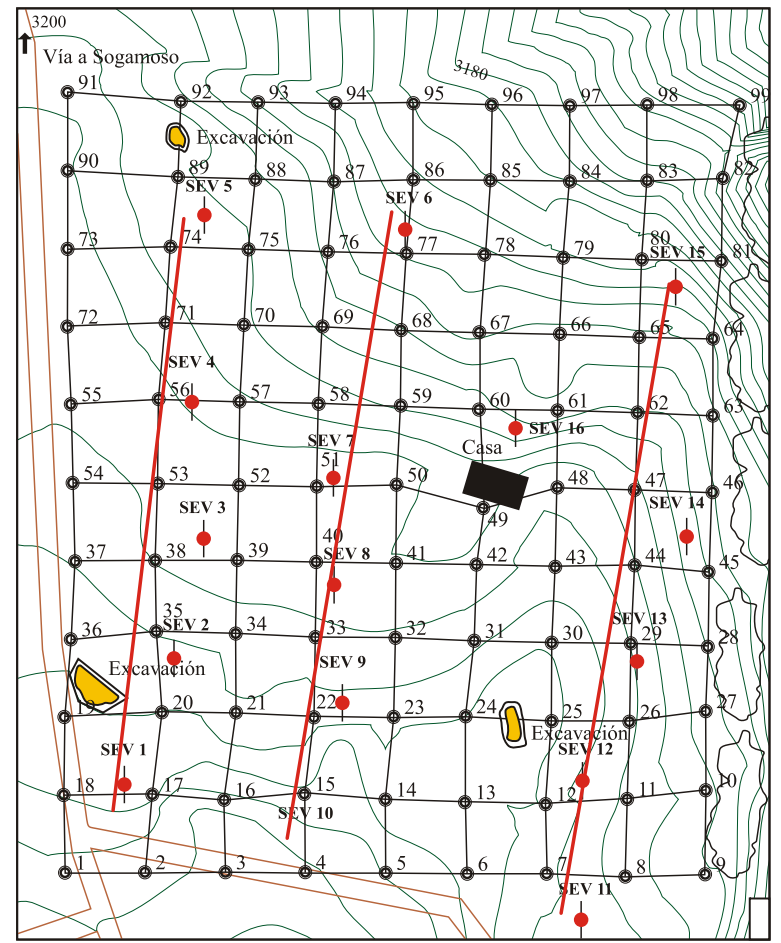

Figura 4. Plano de exploración geofísica.

\subsubsection{Magnetometría}

El levantamiento magnetométrico se llevó a cabo a través de las mediciones con el magnetómetro canadiense que se muestra en la figura 5, en cada uno de los puntos planteados en la malla. A partir de estos datos se confeccionó un mapa (ver figura 6), en donde están representadas las variaciones del campo magnético Ät en nanoTesla.

Adicionalmente fue necesario confeccionar una escala de colores. Las tonalidades de azul oscuro constituyen las zonas de mayor anomalía, con valores entre 180 y 280 nanoTesla, que probablemente corresponden a la manifestación mineral;

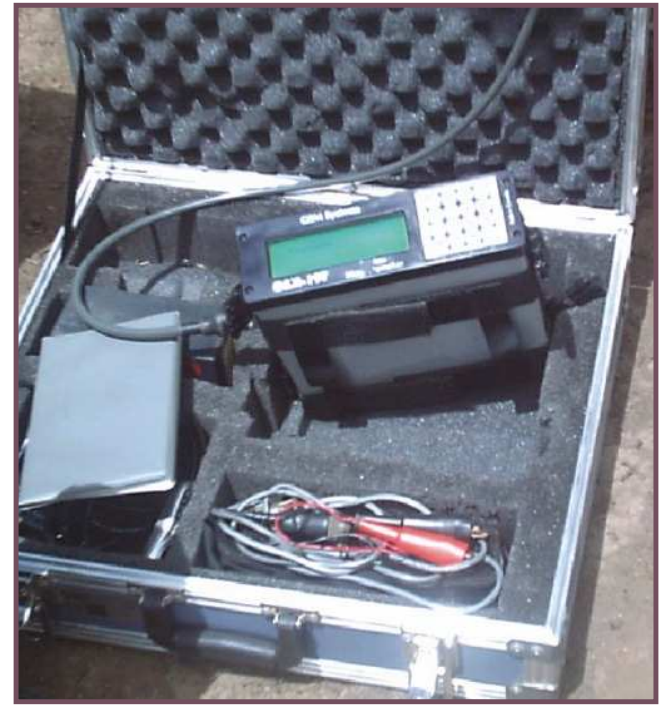

Figura 5. Magnetómetro de fabricación canadiense.

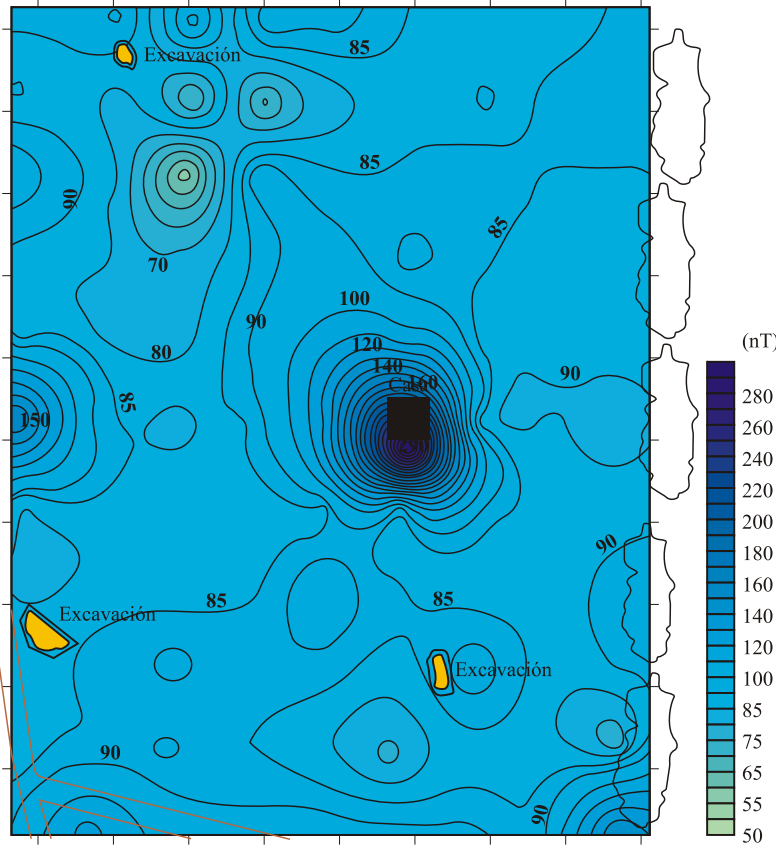

Figura 6. Mapa de anomalía magnética.

mientras que los tonos de amarillo fueron asignados al rango entre 50 y 75 nanoTesla, por ser cifras muy cercanas al valor del campo normal, los cuales fueron asociados a la roca encajante (arcillolita), de la cual se conoce su escasa magnetización. La anterior interpretación se refleja en el mapa geológico (ver figura 7). 


\subsubsection{Cuerpo Cargado}

Este método permitió hallar el valor correspondiente a la resistividad de la mineralización contrastante con el de la roca encajante. Las mediciones se realizan con el equipo DIAPIRVP17, como el que se muestra en la figura 8, y el arreglo de los electrodos para realizar las mediciones de diferencia de potencial e intensidad magnética se esquematiza en la figura 9.

Estos valores de resistividad hallados permitieron confeccionar el mapa de isohomas que se expone en la figura 10. En él se puede leer, en tonalidades de verde, resistividades entre los 850 y $400 \mathrm{ohm}$ por metro, correspondientes presuntamente

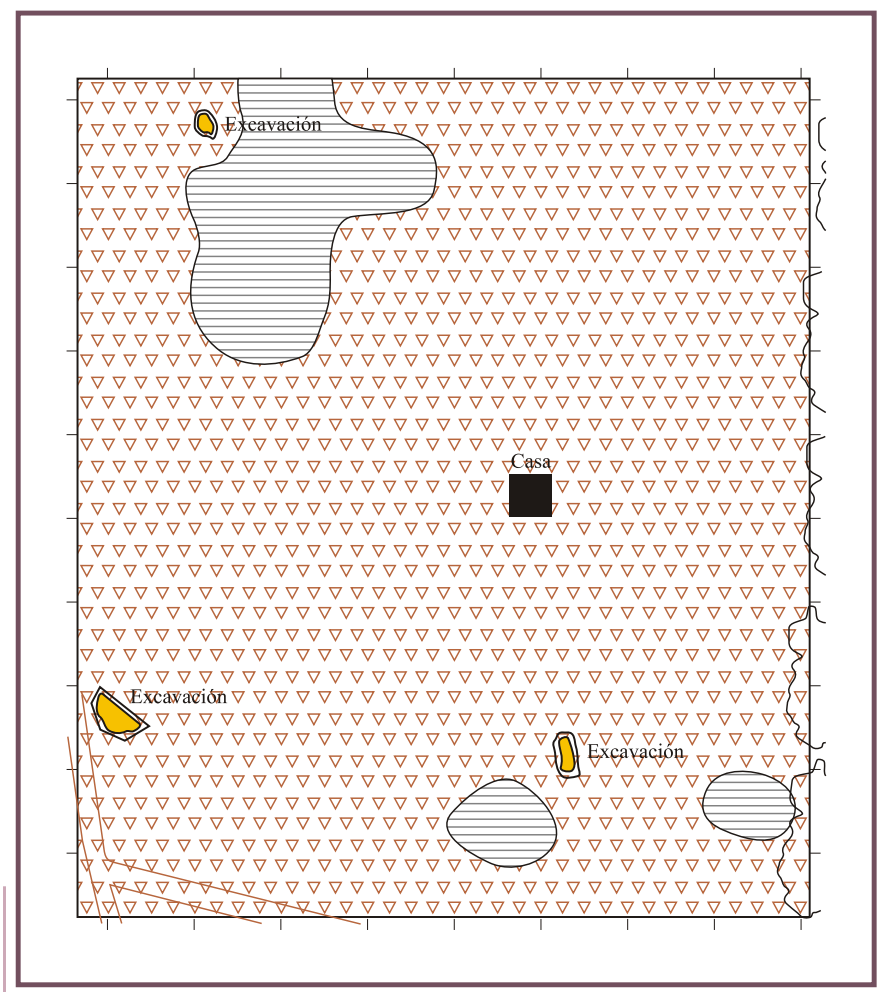

Figura 7. Mapa geológico resultante de la interpretación de la anomalía magnética.

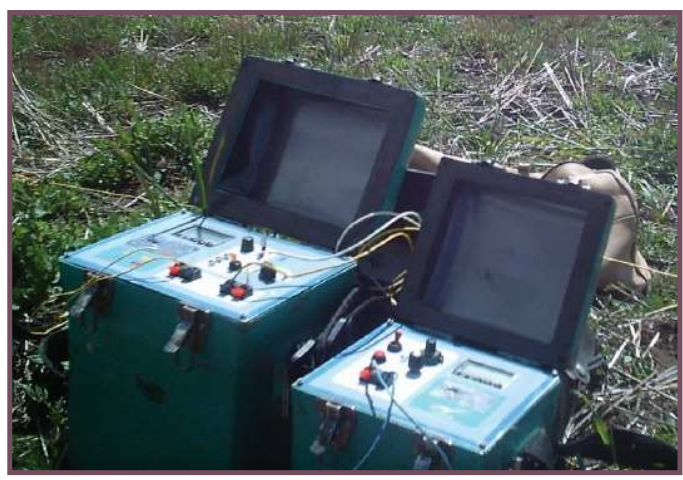

Figura 8. Equipo DIAPIR-VP17. a arcillositas, y en tonalidades de naranja los valores de resistividades entre 350 y $50 \mathrm{ohm}$ por metro, asociados a la manifestación de mineral de hierro por su calidad de material conductor.

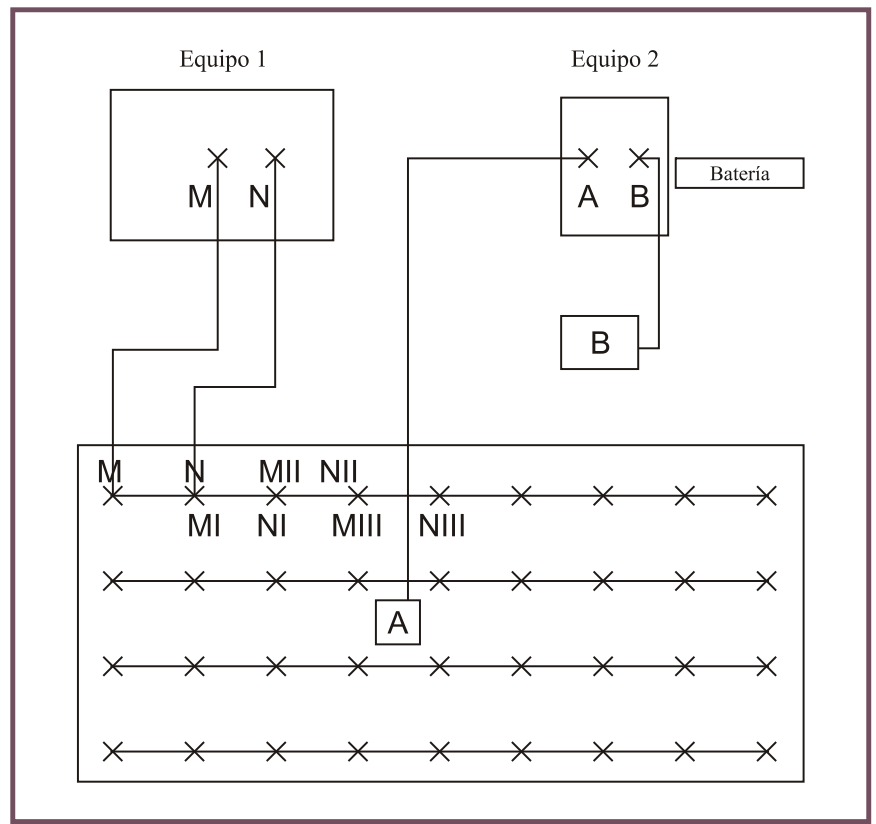

Figura 9. Disposición de los electrodos de medición y alimentación para el método de cuerpo cargado.

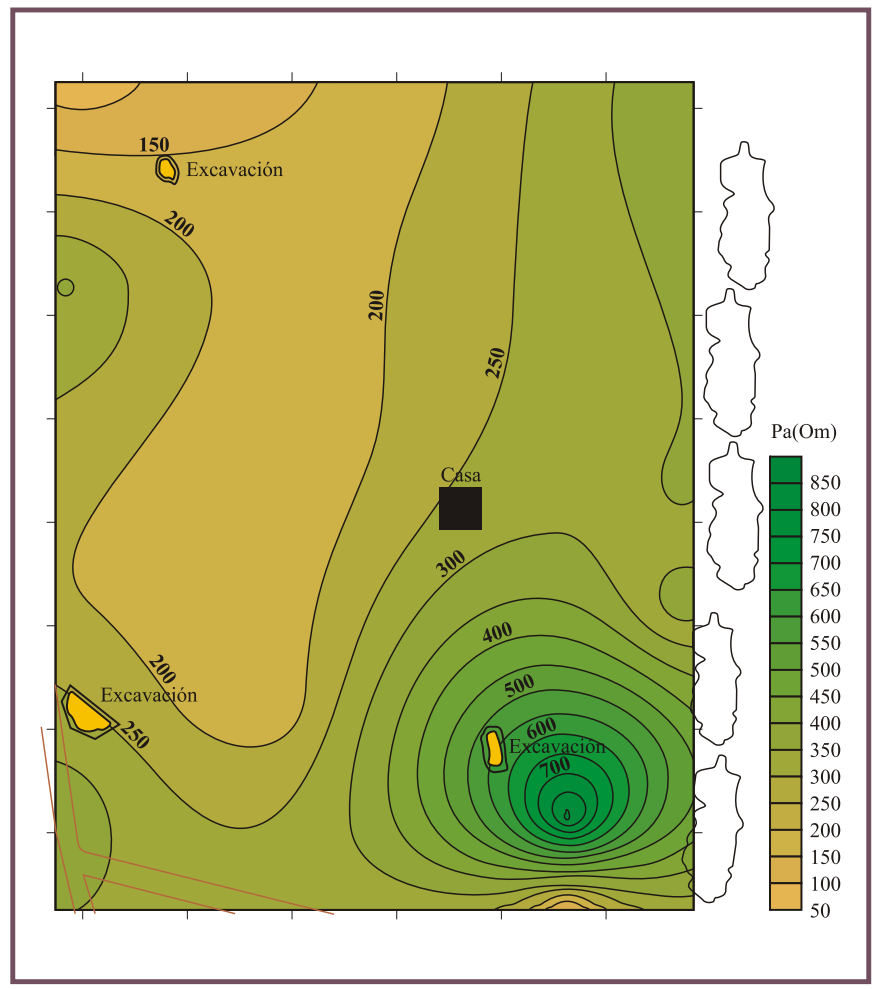

Figura 10. Mapa de isohomas obtenido a partir del método de cuerpo cargado. 
Nuevamente, del análisis realizado se obtiene como resultado un mapa geológico (ver figura 11).

Para un mejor aprovechamiento de la prospección magnética y la aplicación del método del Cuerpo Cargado se realizó un

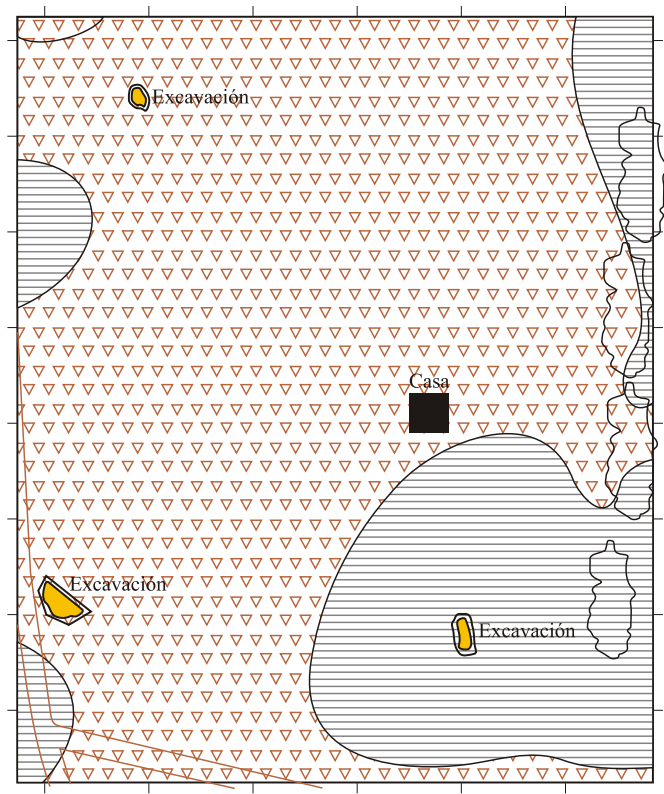

Figura 11. Mapa geológico-geofísico obtenido a partir del método de cuerpo cargado.

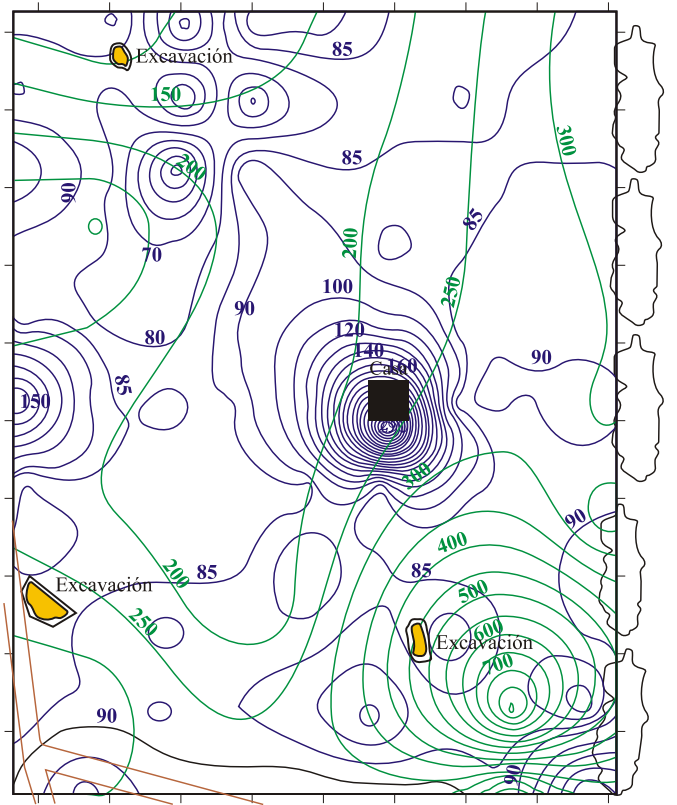

Figura 12. Mapa de interpretación compleja. mapa de interpretación compleja (ver figura 12); en él, los tonos azules corresponden a la anomalía magnética y los tonos verdes, a las isohomas.

El mapa geológico obtenido de este último resultado permitió observar con mayor certeza la distribución del mineral en el área de estudio (ver figura 13).

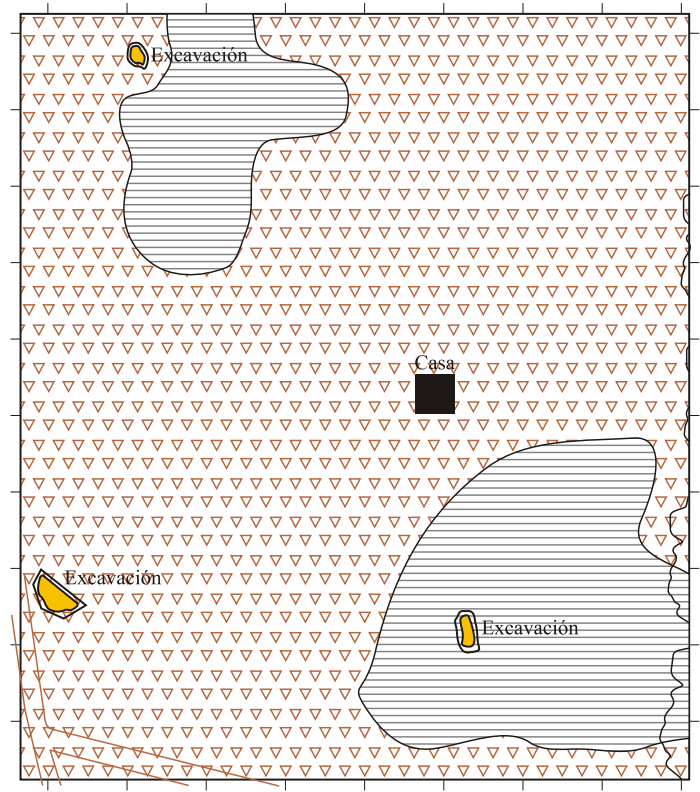

Figura 13. Mapa geológico obtenido a partir del mapa de interpretación compleja.

\subsubsection{Sondeo Eléctrico Vertical}

En esta etapa el trabajo de campo se basa en la adquisición de datos de diferencia de potencial (mv) e intensidad (mA); se utilizó el equipo PASI Italiano en cada uno de los 16 puntos de observación donde la abertura de $\mathrm{AB} / 2$ máxima fue de $80 \mathrm{~m}$. El dispositivo empleado fue el Schlumberger simétrico, en el que se utilizan cuatro electrodos, dos de medición y dos de alimentación, con la disposición mostrada en la figura 14.

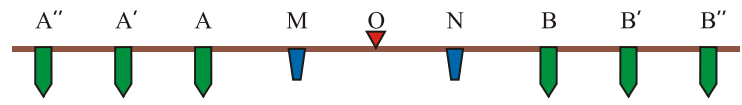

Figura 14. Disposición de los electrodos de medición y alimentación. 
Por medio de este se obtuvieron 16 curvas, como la que se observa en la figura 15, correspondientes a cada uno de los SEV. La geometría predominante de estas fue la tipo $\mathrm{H}$, definida así por los ábacos de Smirnov (1976).

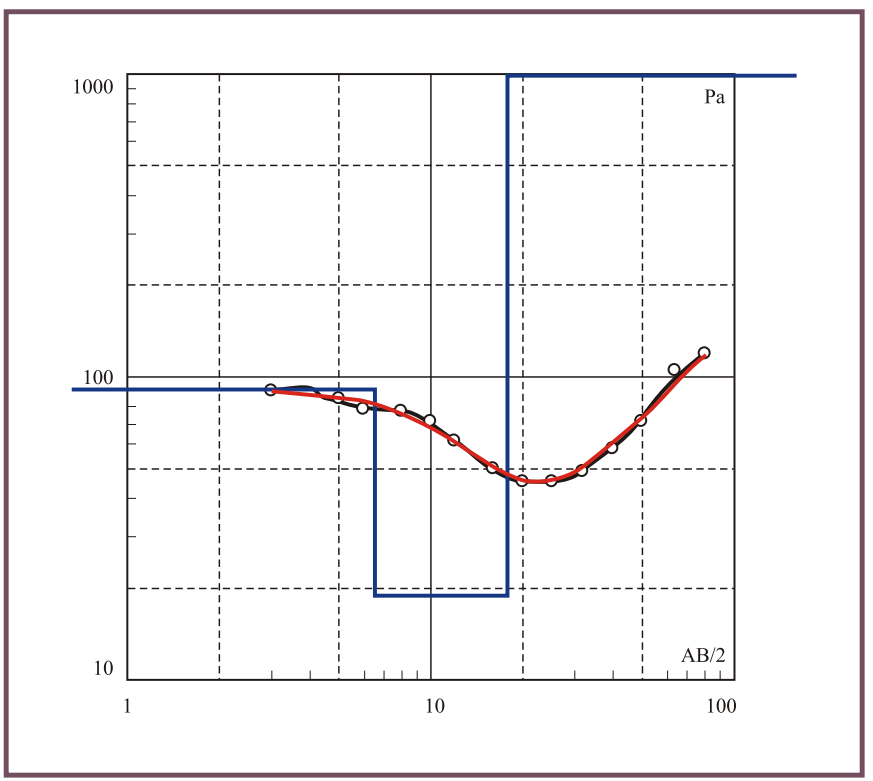

Figura 15. Curva de resistividad aparente generada por el software Ipi2 win.

De la interpretación de cada una de estas curvas se obtuvo una tabla que relaciona la resistividad aparente y la profundidad correspondiente a cada capa (ver tabla 3 ).

Tabla 3. Interpretación de la curva de resistividad aparente.

\begin{tabular}{|c|c|c|}
\hline pa & H & D \\
\hline 91 & 6,54 & 6,54 \\
\hline 19,3 & 11,3 & 17,9 \\
\hline 5856 & & \\
\hline
\end{tabular}

Posteriormente se trazaron 3 perfiles geoeléctricos (ver figura 15) A-A', B-B' y C-C', cuya disposición se observa en la figura 4. Estos perfiles permitieron correlacionar la información de los sondeos y establecer el valor de resistividad aparente para cada litología. El resultado de dicho análisis se encuentra consignado en la tabla 4.
Con esta información se confeccionó el corte geológico correspondiente a cada perfil geoeléctrico (ver figuras 16, 17 y 18), el cual proporcionó la información sobre la disposición del mineral a profundidad. La tonalidad café corresponde al mineral de hierro, representando valores de resistividad muy bajos; en azul se simbolizan las arcillolitas saturadas influenciadas por la presencia de las excavaciones y niveles de agua superficiales; la tonalidad de gris corresponde a la roca encajante aflorante en la zona de estudio, con muy baja saturación y con los valores de resistividad altos.

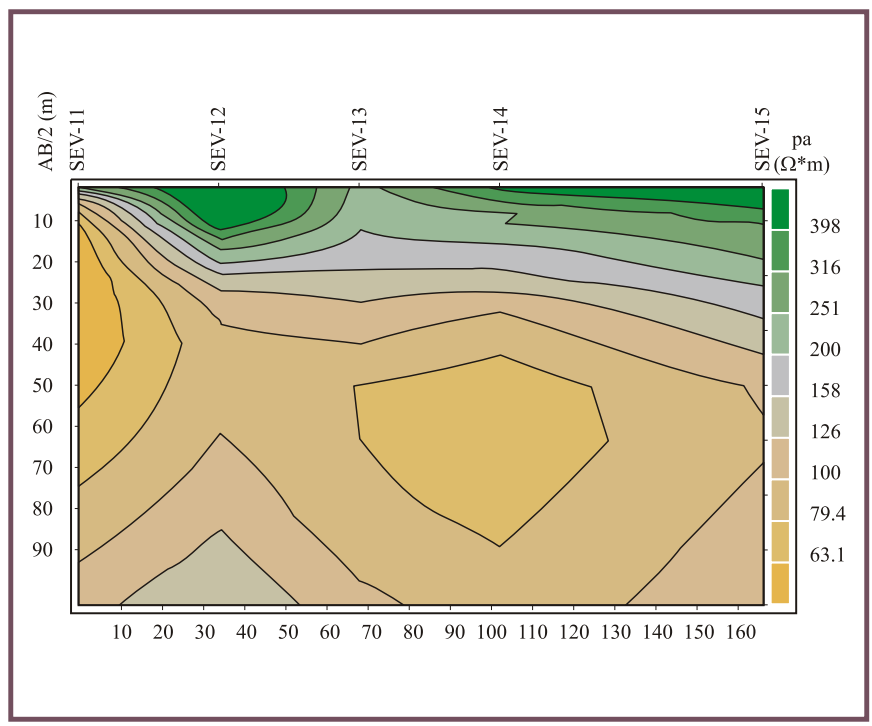

Figura 16. Corte geoeléctrico obtenido en el software Ipi2win.

Tabla 4. Correlación de los valores de resistividad aparente con la litología.

\begin{tabular}{|c|c|c|c|}
\hline $\begin{array}{c}\text { Resistividad } \\
\left(\Omega^{*} \mathbf{m}\right)\end{array}$ & Color & Litología & $\begin{array}{l}\text { Profundidad } \\
\text { (m) }\end{array}$ \\
\hline $215-422$ & & Areillolitas & $3-5$ \\
\hline $150-215$ & & $\begin{array}{c}\text { Arcillolitas } \\
\text { saturadas } \\
\end{array}$ & $5-8$ \\
\hline $50-150$ & & 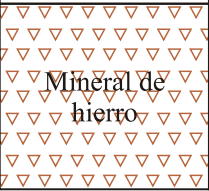 & $8-20$ \\
\hline
\end{tabular}




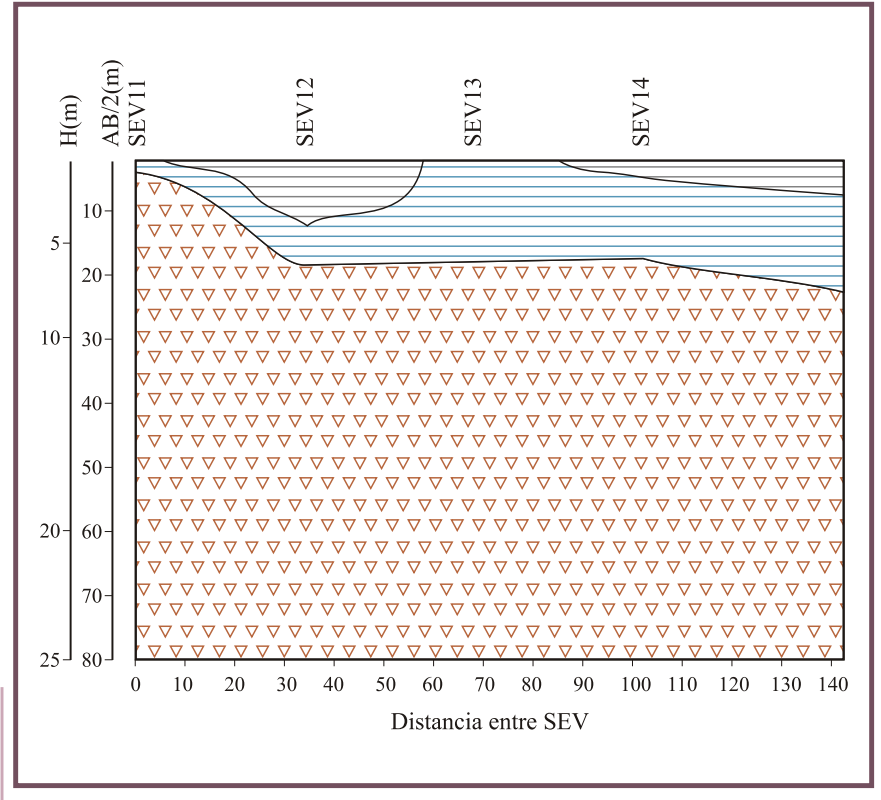

Figura 17. Corte geológico del perfil trazado en dirección A-A'.

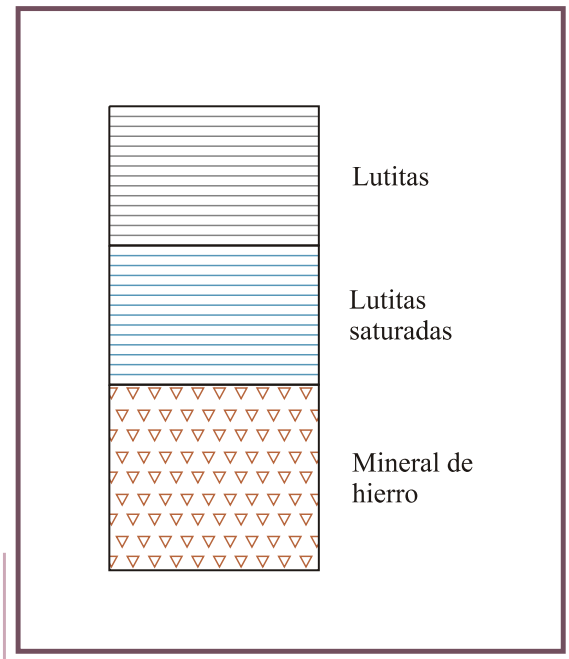

Figura 18. Convenciones utilizadas en el corte geológico.

\section{CONCLUSIONES}

El área más factible para la explotación se encuentra comprendida entre las coordenadas $\mathrm{x}: 1.110 .929 \mathrm{y}: 1.115 .167 \mathrm{y}$ $\mathrm{x}: 1.110 .948 \mathrm{y}: 1.115 .312$, que es por donde pasa el perfil número 1 (ver figura 4). De allí se conoce que el mineral está a una profundidad de $8 \mathrm{~m}$ en adelante, no está influenciado por la vivienda y queda cerca a la carretera para facilitar su transporte.

Los métodos geofísicos ayudaron a determinar que el área estudiada corresponde a una zona de mineralización de hierro.
Esta mineralización no ocurre en forma masiva, por lo que en el mapa de anomalía magnética no fue posible hallar profundidades, tan solo lograr diferenciar las zonas más anómalas para aplicar otros métodos.

Aunque todos los métodos son importantes, se destaca el aporte realizado por el método del sondeo eléctrico vertical, ya que fue el único que permitió determinar aproximadamente la profundidad a la cual se encontraba el mineral de hierro.

El estudio petrográfico, por medio del contador de puntos, fue fundamental para determinar una composición del mineral encontrado. Siendo satisfactorio, ya que la gohetita se encuentra en $52 \%$.

El conocimiento geológico y la experiencia en la interpretación son factores determinantes a la hora de correlacionar los valores dados por los métodos geofísicos.

Las excavaciones realizadas en el área de estudio llevaron a la degradación del paisaje; esto se habría podido evitar con la realización, por parte de las empresas interesadas, de campañas de exploración geofísica previas a las excavaciones. Los costos de la exploración son mucho más económicos que las excavaciones a ciegas y evitan el daño al medioambiente.

\section{RECOMENDACIONES}

Evaluar el área con un cuarto método que permita determinar profundidades para evaluar la eficacia del sondeo eléctrico vertical.

Evaluar los resultados obtenidos con los diferentes software, de tal manera que se pueda establecer un patrón, como es el caso de la resistividad, para que así los resultados sirvan a investigaciones futuras.

Hacer un estudio petrográfico de mayor exactitud; se recomienda el ensayo de difracción de rayos $\mathrm{X}$.

Realizar un estudio de tipo ambiental para determinar la factibilidad de explotar el mineral de hierro encontrado.

\section{REFERENCIAS}

Baterman, Allan (1951). La formación de depósitos minerales. Jhons and Sons.

Dobrin, M. B. (1961). Introducción a la prospección geofísica. Barcelona: Omega.

Danko, I. (1972). Geoelectricidad I. La Habana: Escuela de ingeniería geofísica.

Hernández, Hugo Alfonso (1973). Petrofísica. La Habana: Editorial pueblo y educación.

Ladoo, R y Myers, W. (1951). Minerales no metálicos. New York: Mc. Graw Hill.

Logachev, A y Zajarov, V. (1978). Exploración magnética. Barcelona: Reverté S.A.

Orellana, Ernesto (1972). Prospección geoeléctrica en corriente continua. Madrid: Paraninfo. 